\title{
Stratified Kolmogorov flow. Part 2
}

\author{
By N. J. BALMFORTH ${ }^{1}$ AND Y.-N. YOUNG ${ }^{2}$ \\ ${ }^{1}$ Departments of Mathematics and Earth \& Ocean Sciences, University of British Columbia, 1984 \\ Mathematics Road, Vancouver, BC, Canada V6T 1 Z2 \\ ${ }^{2}$ Department of Mathematical Sciences, New Jersey Institute of Technology, Newark, NJ 07102, USA
}

(Received 11 February 2004 and in revised form 26 September 2004)

Forced stratified flows are shown to suffer two types of linear long-wave instability: a 'viscous' instability which is related to the classical instability of Kolmogorov flow, and a 'conductive instability', with the form of a large-scale, negative thermal diffusion. The nonlinear dynamics of both instabilities is explored with weakly nonlinear theory and numerical computations. The introduction of stratification suppresses the viscous instability, but also makes it subcritical. The second instability arises with stronger stratification and creates a prominent staircase in the buoyancy field; the steps of the staircase evolve over long timescales by approaching one another, colliding and merging (coarsening the staircase).

\section{Introduction}

Stratified shear flows arise frequently in geophysical and astrophysical fluid dynamics. A central issue in such contexts is understanding how eddying unsteady motion arises from a steady flow or forcing, and how that motion can re-arrange and transport the fluid properties. In this article, we continue an exploration of a particular model problem in which the dynamics is accessible to an unusual degree of analysis. More specifically, we study the fully stratified version of the so-called Kolmogorov flow, which was originally advocated as a convenient theoretical construct for understanding unstratified shear flow dynamics and the transition to turbulence. Instabilities of Kolmogorov flow exhibit the property of inverse cascade: although instabilities can be seeded on moderate length scales, energy is continually transferred via nonlinear mechanisms to longer lengthscales. In our previous article (Balmforth \& Young 2002), we showed how the cascade is arrested by relatively weak stratification. This arrest is also implicit in much of the exploration described in the current article. However, it does not provide our main focus, which lies in a different direction.

Laboratory experiments and oceanic observations have both revealed that flows in stably stratified fluids can generate 'staircases' of well-mixed layers separated by sharp interfaces. In the laboratory, staircases have been created by dragging grids or bars through tanks of salt-stratified water (Park, Whitehead \& Gnanadeskian 1996; Holford \& Linden 2000); in the ocean, mixing by the motions of the ever turbulent environment is assumed to have the same effect (Schmitt 1994). Small-scale fingering instability due to double diffusion is also thought to create large-scale staircases without externally driven flows (Radko 2003), and turbulent thermohaline convection has been seen to generate stacked layers in the laboratory and solar ponds (e.g. Turner 1985). Although it has never been shown explicitly, it is commonly assumed that a turbulent flow field is an essential ingredient in the layering problem. That is, that the Reynolds number of the mixing flow must be very large. Based on this premise, several authors constructed crude models of turbulent stratified fluids and 
thereby rationalized the layering process (Phillips 1972; Posmentier 1977; Balmforth, Llewellyn Smith \& Young 1998). These models typically rely on simple, sometimes empirical, parameterizations of turbulent transport, and formulate a non-monotonic relation between the density flux and the density gradient (the 'flux-gradient relation'). The underlying notion is that wherever the flux decreases with the gradient, the stratification is unstable and small fluctuations will seed the growth of sharp steps via negative diffusion.

One of our purposes in the present article is to show that staircases can also result for much lower Reynolds numbers, when the mixing flows are laminar. This opens up the problem to analytical explorations based on the governing equations of fluid mechanics, rather than crude turbulence parameterizations. In particular, by using the method of multiple scales, we establish that instability can occur in the form of negative diffusion, and determine the flux-gradient relation explicitly in the vicinity of the onset of instability. Staircases can then be predicted to occur; we examine the robustness of layering within this formulation. Our analysis is similar to that used to compute eddy diffusivities in homogeneous fluid (Gama, Vergolassa \& Frisch 1994), and there are analogies with stability theories of Rossby waves (Lorenz 1972) and internal gravity waves (Drazin 1978; Kurgansky 1979, 1980; Lombard \& Riley 1996) which have applications to atmospheric dynamics and to oceanic mixing (Thorpe 1994).

Our analysis proceeds by way of multiple scales, assuming that instability arises on a much longer spatial scale than the intrinsic lengthscale of the steady flow pattern that is set up by a suitable body forcing of the fluid (the Kolmogorov flow). This analysis detects linear, long-wave instability $(\S 2)$ which we then continue on to explore at the finite-amplitude level using weakly nonlinear techniques (section $\S 3$ ) and numerical computation (section $\S 4$ ).

\section{Formulation and linear theory}

\subsection{Governing equations}

We begin with the basic equations for a forced fluid in the Boussinesq approximation. After introducing a streamfunction, $\psi(x, z, t)$, and the buoyancy field, $b(x, z, t)$ (representing an agent such as temperature or salinity), which describe the deviation from the motionless (linearly) stratified state, these equations are

$$
\begin{gathered}
\nabla^{2} \psi_{t}+J_{x, z}\left(\psi, \nabla^{2} \psi\right)=b_{x}+\nu \nabla^{4} \psi-\nu \nabla^{4} \varphi, \\
b_{t}+J_{x, z}(\psi, b)+N^{2} \psi_{x}=\kappa \nabla^{2} b,
\end{gathered}
$$

where $\varphi$ represents the forced source of vorticity,

$$
J_{r, s}(f, g)=f_{r} g_{s}-f_{s} g_{r}
$$

is the Jacobian of the functions $f$ and $g$ with respect to the coordinates $r$ and $s, v$ is the viscosity, $\kappa$ the conductivity, and $N^{2}$ the buoyancy frequency arising from the background linear stratification. For practical purposes, we take

$$
\varphi=\varphi_{0} \sin (k x-m z),
$$

where $\varphi_{0}$ is the amplitude of the forcing, and the wavenumbers, $(k, m)$, determine the tilt with respect to the vertical. This forcing generates a steady equilibrium flow of the form $(u, w)=\Psi(m, k) \cos (k x-m z)$, where $\Psi$ is a constant. We impose periodic boundary conditions in the horizontal, and delay discussion of the vertical boundary conditions until later. 
We place the equations in a non-dimensional form using units given by the forcing; that is, the lengthscale, $K^{-1}$, and the timescale, $\varphi_{0} K^{2}$, where $K^{2}=k^{2}+m^{2}$. We define $x^{\prime}=K x, z^{\prime}=K z, t^{\prime}=\varphi_{0} K^{2} t, \psi^{\prime}=\psi / \varphi_{0}$ and $b^{\prime}=K^{3} \varphi_{0}^{2} b$. On substitution into (2.1)(2.2), and discarding the primes, we arrive at

$$
\begin{gathered}
\nabla^{2} \psi_{t}+J_{x, z}\left(\psi, \nabla^{2} \psi\right)=b_{x}+R e^{-1}\left(\nabla^{4} \psi-\cos \phi\right), \\
b_{t}+J_{x, z}(\psi, b)+\beta \psi_{x}=P e^{-1} \nabla^{2} b,
\end{gathered}
$$

where $\phi=x \cos \theta+z \sin \theta$, with $m=K \sin \theta$ and $k=K \cos \theta$, the dimensionless groups, $R e=\varphi_{0} / \nu$ and $P e=\varphi_{0} / \kappa$, denote the Reynolds and Péclet numbers, and $\beta=N^{2} /\left(\varphi_{0}^{2} K^{4}\right)$ is a stratification parameter somewhat like a Richardson number.

\subsection{Multiple-scale expansion}

We introduce

$$
\partial_{t} \rightarrow \epsilon^{2} \partial_{T}, \quad \partial_{z} \rightarrow \partial_{z}+\epsilon \partial_{Z},
$$

where $T \equiv \epsilon^{2} t$ and $Z \equiv \epsilon z$ denote a slow timescale and a long lengthscale (a corresponding long scale for $x$ turns out to be not necessary because the first solvability conditions that one then encounters demand that there be no variation on such a scale, at least for order-one $\beta$ ). With these rescalings

$$
\begin{aligned}
\epsilon^{2}\left[\partial_{x}^{2}+\left(\partial_{z}+\epsilon \partial_{Z}\right)^{2}\right] \psi_{T}+ & J_{x, z}\left(\psi, \psi_{x x}+\left(\partial_{z}+\epsilon \partial_{Z}\right)^{2} \psi\right)+\epsilon J_{x, Z}\left(\psi, \psi_{x x}+\left(\partial_{z}+\epsilon \partial_{Z}\right)^{2} \psi\right) \\
& =b_{x}+R e^{-1}\left[\partial_{x}^{2}+\left(\partial_{z}+\epsilon \partial_{Z}\right)^{2}\right]^{2} \psi-R e^{-1} \cos \phi, \\
\epsilon^{2} b_{T}+J_{x, z}(\psi, b)+ & \epsilon J_{x, Z}(\psi, b)+\beta \psi_{x}=P e^{-1}\left[\partial_{x}^{2}+\left(\partial_{z}+\epsilon \partial_{Z}\right)^{2}\right] b
\end{aligned}
$$

Over the shorter spatial scales, $(x, z)$, we look for solutions that have the same periodicity as the forcing; these patterns are modulated on the long spatial scale $Z$ and over the slow time.

It is useful to quote the averages over the $(x, z)$-scales:

$$
\epsilon \bar{\psi}_{T}+\overline{\psi_{x} \psi_{Z}}=\epsilon R e^{-1} \bar{\psi}_{Z Z}, \quad \epsilon \bar{b}_{T}+\left(\overline{\psi_{x} b}\right)_{Z}=\epsilon P e^{-1} \bar{b}_{Z Z}
$$

where the bar denotes the average over a spatial period of the forcing, and we have integrated the first relation twice in $Z$, assuming the integration constants vanish by virtue of the boundary conditions in $Z$.

We now introduce the asymptotic sequences,

$$
\psi=\psi_{0}+\epsilon \psi_{1}+\cdots, \quad b=b_{0}+\epsilon b_{1}+\cdots,
$$

and collect together terms of like order. At order one,

$$
\begin{gathered}
\operatorname{Re} b_{0 x}+\left(\partial_{x}^{2}+\partial_{z}^{2}\right)^{2} \psi_{0}-\operatorname{Re} J_{x, z}\left(\psi_{0}, \psi_{0 x x}+\psi_{0 z z}\right)=\cos \phi, \\
\beta P e \psi_{0 x}-\left(\partial_{x}^{2}+\partial_{z}^{2}\right) b_{0}+P e J_{x, z}\left(\psi_{0}, b_{0}\right)=0,
\end{gathered}
$$

with solution,

$$
\psi_{0}=\bar{\psi}_{0}(Z, T)+\frac{\cos \phi}{1+G}, \quad b_{0}=\bar{b}_{0}(Z, T)+\frac{\beta P e \sin \phi \cos \theta}{1+G},
$$

where $G=\beta R e P e \cos ^{2} \theta$.

At the following order:

$$
\operatorname{Re} b_{1 x}+\left(\partial_{x}^{2}+\partial_{z}^{2}\right)^{2} \psi_{1}=-\frac{\operatorname{Re} \bar{\psi}_{0 Z} \sin \phi \cos \theta}{1+G}+N_{1},
$$




$$
\beta P e \psi_{1 x}-\left(\partial_{x}^{2}+\partial_{z}^{2}\right) b_{1}=\frac{\beta P e^{2} \bar{\psi}_{0 Z} \cos \phi \cos ^{2} \theta}{1+G}+\frac{P e \bar{b}_{0 Z} \sin \phi \cos \theta}{1+G}+N_{2},
$$

where $N_{1}$ and $N_{2}$ are nonlinear terms that vanish for the solution,

$$
\begin{gathered}
\psi_{1}=\bar{\psi}_{1}(Z, T)+\frac{R e \cos \theta}{(1+G)^{2}}\left[\left(\beta P e^{2} \cos ^{2} \theta-1\right) \bar{\psi}_{0 Z} \sin \phi+P e \bar{b}_{0 Z} \cos \theta \cos \phi\right], \\
b_{1}=\bar{b}_{1}(Z, T)+\frac{P e \cos \theta}{(1+G)^{2}}\left[(R e+P e) \beta \bar{\psi}_{0 Z} \cos \theta \cos \phi-\bar{b}_{0 Z} \sin \phi\right] .
\end{gathered}
$$

We substitute these solutions into the average equations to find two diffusion equations,

$$
\begin{aligned}
\operatorname{Re} \bar{\psi}_{0 T} & =\left[1-\frac{\operatorname{Re}^{2}(1-\sigma G) \cos ^{2} \theta}{2(1+G)^{3}}\right] \bar{\psi}_{0 Z Z}, \\
\operatorname{Pe} \bar{b}_{0 T} & =\left[1-\frac{P e^{2}(G-1) \cos ^{2} \theta}{2(1+G)^{3}}\right] \bar{b}_{0 Z Z},
\end{aligned}
$$

where $\sigma=v / \kappa$ is the Prandtl number.

\subsection{Critical conditions}

The diffusivities in (2.18)-(2.19) are not positive definite. Indeed, for certain choices of the parameters, these quantities may become negative, signifying a long-scale instability. Each equation provides an instability condition:

$$
1<\frac{R e^{2}(1-\sigma G) \cos ^{2} \theta}{2(1+G)^{3}}, \quad 1<\frac{P e^{2}(G-1) \cos ^{2} \theta}{2(1+G)^{3}} .
$$

At this stage, we observe that the only effect of $\theta$ is to rescale the Reynolds and Péclet numbers, and so the inclination of the forcing has a minor effect on the linear, long-wave dynamics. For brevity we therefore set $\theta=0$ hereafter.

Mathematically, it is convenient to select $\operatorname{Re}, \sigma$ and $G$ as the governing parameters of the problem. We may then translate the conditions in (2.20) into the critical Reynolds numbers,

$$
R e>R e_{1}=\frac{\sqrt{2}(1+G)^{3 / 2}}{(1-\sigma G)^{1 / 2}}, \quad R e>R e_{2}=\frac{\sqrt{2}(1+G)^{3 / 2}}{\sigma(G-1)^{1 / 2}} .
$$

If $G=0, R e_{1} \rightarrow \sqrt{2}$ whereas $R e_{2}$ ceases to exist. The former is the instability threshold of the usual Kolmogorov instability (Meshalkin \& Sinai 1960), which can be seen from (2.18) to result from a negative effective viscosity. As shown in figure 1, the instability becomes modified by stratification, and even removed, when $G$ is increased from zero. Thus, $R e_{1}$ characterizes a familiar long-wave instability that we refer to as 'viscous'. The nonlinear dynamics of the weakly stratified viscous instability was considered by Young (1999) and Balmforth \& Young (2002).

The other critical threshold, $R e_{2}$, corresponds to a second type of instability which (2.19) reveals to result from negative conduction. This second mode of instability appears only at higher stratification ( $G$ or $\beta$ ), and we refer to it as 'conductive'. The two instabilities typically appear in different parts of parameter space, although they can be coincident when $\sigma<1$ (see figure 1). The instabilities appear simultaneously for $R e_{1}=R e_{2}$, which demands that

$$
G=\frac{1+\sigma^{2}}{\sigma(1+\sigma)}
$$




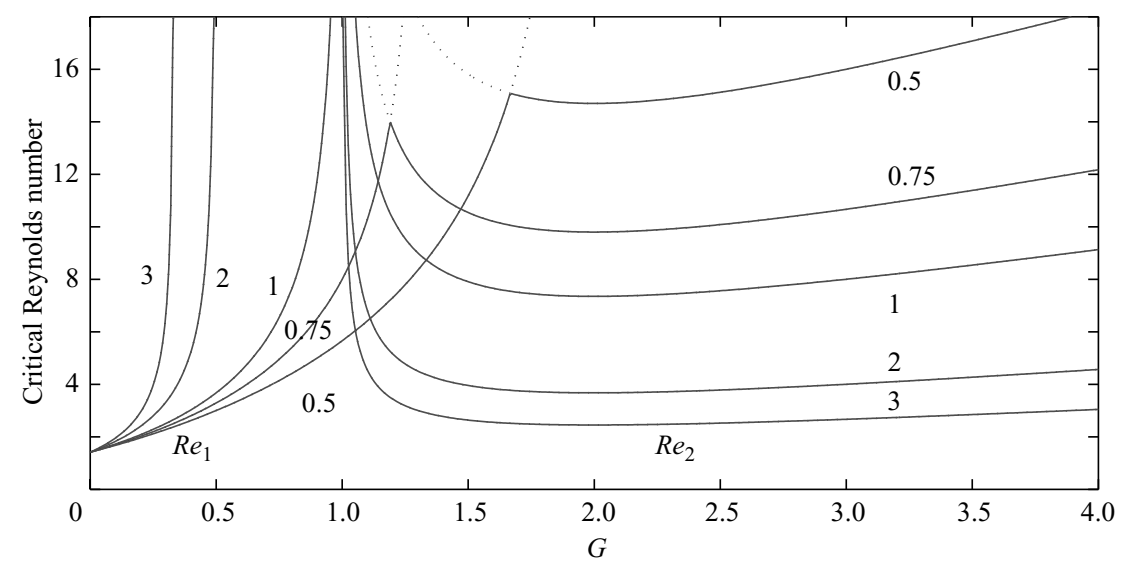

Figure 1. Critical Reynolds numbers, $\operatorname{Min}\left(R e_{1}, R e_{2}\right)$, against $G$ for $\theta=0$ and several values of Prandtl number (as labelled).

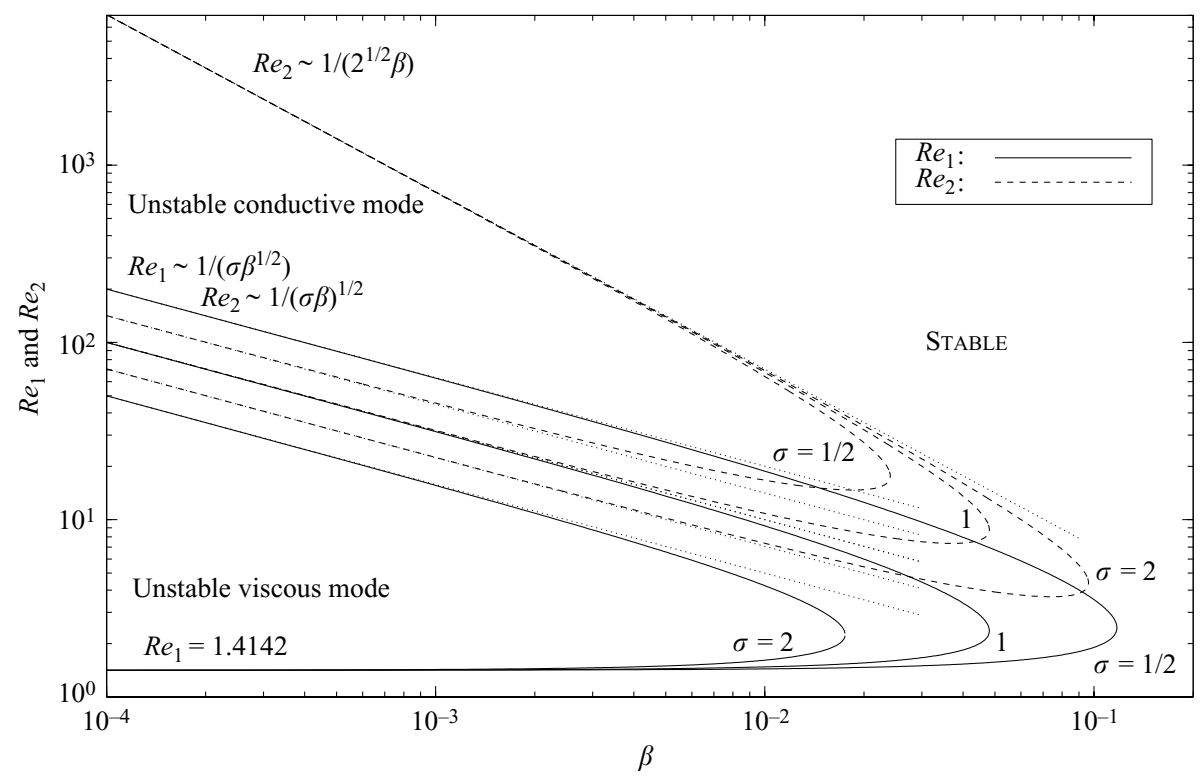

FIgURE 2. The critical Reynolds numbers $R e_{1}$ and $R e_{2}$ against the stratification parameter, $\beta$, for $\sigma=1 / 2,1$ and 2 . The limiting thresholds as $\beta \rightarrow 0$ are also indicated.

In cases in which $\beta$ is the dimensionless parameter that can be prescribed, $G$ is not a suitable parameter to parameterize the critical thresholds of the system because $G=\sigma \beta R e^{2}$. However, eliminating $G$ in favour of $\beta$ complicates the critical conditions, and $R e_{1}$ and $R e_{2}$ cannot be found in closed form. Instead, we show sample critical thresholds computed numerically against $\beta$ for different Prandtl numbers in figure 2. This figure illustrates how stratification stabilizes the viscous instability at large Reynolds number, and completely removes instability for any Reynolds number beyond a $\sigma$-dependent value. The instability window of the conductive mode is similar, but typically lies at higher Reynolds number and only overlaps the region of viscous instability for $\sigma<1$. The values of $\beta$ and Reynolds number for which the two instabilities disappear entirely (that is, the values at the nose of the stability boundaries in figure 2) are shown against Prandtl number in figure 3 . Note that the 

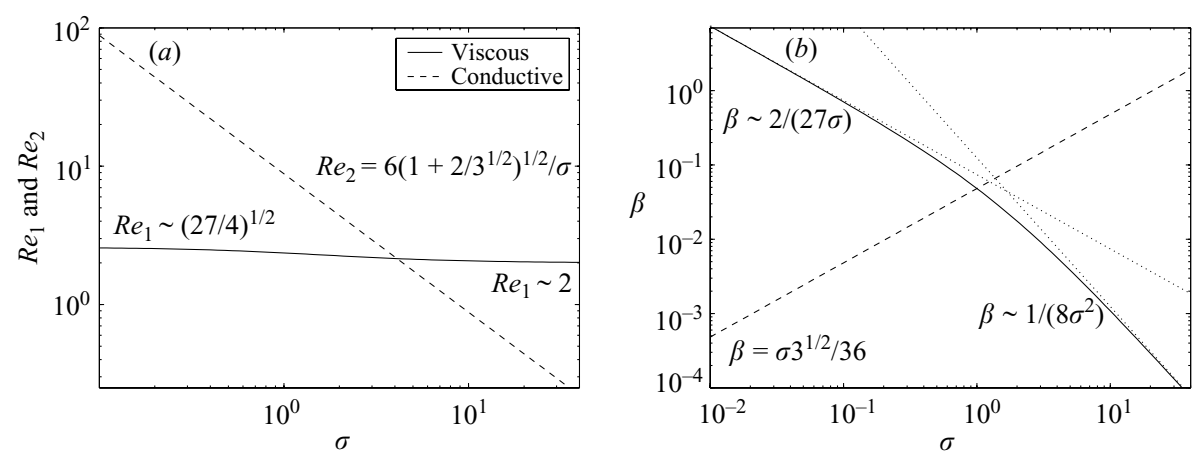

FIgURE 3. (a) The Reynolds numbers, $R e_{1}$ and $R e_{2}$, and $(b)$ stratification parameter, $\beta$, plotted against $\sigma$ at the 'nose' of the long-wave stability boundaries (i.e. the values of $R e_{j}$ and $\beta$ for which instability disappears entirely). The limiting values for $\sigma \rightarrow 0$ and $\infty$ are indicated.

stabilizing effect of stratification, and specifically the removal of the instability for any Reynolds number beyond a critical value of $\beta$, is reminiscent of the celebrated Richardson number criterion. However, the basic flow is in the direction of gravity here, and the critical threshold in $\beta$ has a novel dependence on Prandtl number, as illustrated in figure 3 .

Note that the theory identifies only long-wave instabilities. However, instabilities with finite wavenumber are also possible, and these would lead to more windows of instability elsewhere in parameter space. Some confidence that long waves are responsible for instability comes from the numerical solutions of $\S 4$ at isolated parameter values, although these computations also uncover other instabilities. A more systematic approach would entail a detailed numerical exploration of the linear stability problem for arbitrary wavenumber.

\section{Nonlinear theory}

We next demonstrate that layering is expected in the mildly nonlinear stages of the instability discussed above. We proceed by deriving a Cahn-Hilliard equation through a weakly nonlinear asymptotic expansion, an equation that is known to possess solutions in the form of layers. We perform this construction for both the viscous and conductive instabilities (taking $\theta=0$ ).

For both instabilities, we again introduce the long scale, $Z=\epsilon z$, and rescale time, but in a slightly different way: $\partial_{t} \rightarrow \epsilon^{4} \partial_{\tau}\left(\tau=\epsilon^{4} t\right)$. Then, the governing equations become

$$
\begin{gathered}
\epsilon^{4}\left(\partial_{x}^{2}+\epsilon^{2} \partial_{Z}^{2}\right) \psi_{\tau}+\epsilon\left(\psi_{x} \psi_{x x Z}-\psi_{Z} \psi_{x x x}\right)+\epsilon^{3}\left(\psi_{x} \psi_{Z Z Z}-\psi_{Z} \psi_{x Z Z}\right) \\
=b_{x}+R e^{-1}\left(\partial_{x}^{2}+\epsilon^{2} \partial_{Z}^{2}\right)^{2} \psi-R e^{-1} \cos x, \\
\epsilon^{4} b_{\tau}+\epsilon\left(\psi_{x} b_{Z}-\psi_{Z} b_{x}\right)+\beta \psi_{x}=P e^{-1}\left(\partial_{x}^{2}+\epsilon^{2} \partial_{Z}^{2}\right) b
\end{gathered}
$$

\subsection{Weak conductive instability}

To derive the Cahn-Hilliard model in the conductive case, we begin with a flow on the brink of instability, and then kick the system into the unstable regime by slightly modifying the parameter values. In particular, we focus on the specific parameter choices,

$$
\beta=3 R e^{-1} \sqrt{6}\left(2+\epsilon G_{1}\right), \quad P e^{-1}=\frac{1}{3 \sqrt{6}}+\epsilon^{2} \kappa_{2} .
$$


That is, we tune both $\beta$ and $P e$, treating $R e$ as a free parameter. The two choices in (3.3) are necessary for reasons that will be discussed later; essentially, we obtain the Cahn-Hilliard equation only at a codimension-two point where the coefficient of the leading quadratic nonlinear term is forced to vanish.

We begin again from the governing equations in the forms (3.1)-(3.2), but now choose the leading-order solution,

$$
\psi_{0}=\frac{1}{3} \cos x, \quad b_{0}=B(Z, \tau)+\frac{2}{3 R e} \sin x .
$$

The significance of this choice is that in (2.18)-(2.19) there are two long-wave modes evolving on the $t \sim \epsilon^{-2}$ timescale: a viscous mode and a conductive mode. Only the second of these modes is marginally stable for $G_{1}=\kappa_{2}=0$, and therefore evolves even more slowly on the $t \sim \epsilon^{-4}$ time. The viscous mode, on the other hand, at this point is more heavily damped. As a result, we assume that the mode decays to low amplitude before the conductive mode begins to grow (which does not, in fact, always remain true as shown by computations reported below), leading us to include only the slow mode, $B(Z, \tau)$.

At the next order, we find

$$
\operatorname{Re} b_{1 x}+\psi_{1 x x x x}=0, \quad 2 \psi_{1 x}-\operatorname{Re} b_{1 x x}=\frac{1}{3}\left(3 \operatorname{Re} \sqrt{6} B_{Z}+G_{1}\right) \sin x .
$$

We take

$$
\psi_{1}=-\left(\frac{\operatorname{Re} \sqrt{6}}{3} B_{Z}+\frac{G_{1}}{9}\right) \cos x, \quad b_{1}=\left(\frac{\sqrt{6}}{3} B_{Z}+\frac{G_{1}}{9 \operatorname{Re}}\right) \sin x .
$$

At order $\epsilon^{2}$,

$$
\begin{gathered}
\operatorname{Re} b_{2 x}+\psi_{2 x x x x}=0, \\
2 \psi_{2 x}-\operatorname{Re} b_{2 x x}=-3 \operatorname{Re} B_{Z Z} \cos 2 x-\left[\left(\frac{G_{1}}{3}+\sqrt{6} \operatorname{Re} B_{Z}\right)^{2}+2 \kappa_{2} \sqrt{6}\right] \sin x,
\end{gathered}
$$

which we solve with

$$
\begin{aligned}
& \psi_{2}=\frac{1}{3}\left[\left(\frac{G_{1}}{3}+\sqrt{6} \operatorname{Re} B_{Z}\right)^{2}+2 \kappa_{2} \sqrt{6}\right] \cos x-\frac{R e}{12} B_{Z Z} \sin 2 x, \\
& b_{2}=-\frac{1}{3 \operatorname{Re}}\left[\left(\frac{G_{1}}{3}+\sqrt{6} \operatorname{Re} B_{Z}\right)^{2}+2 \kappa_{2} \sqrt{6}\right] \sin x-\frac{2}{3} B_{Z Z} \cos 2 x .
\end{aligned}
$$

The third-order equations and their solution proceed in much the same way. For brevity, key formulae are relegated to the appendix. Finally, we insert the solutions for $\psi$ and $b$ into the horizontal averages of the governing system to arrive at the amplitude equation,

$$
B_{\tau}=2\left(\kappa_{2}+\frac{G_{1}^{2} \sqrt{6}}{108}\right) B_{Z Z}-\left(\frac{13 \sqrt{6}}{72}+\frac{R e}{108}\right) B_{Z Z Z Z}+\frac{G_{1} R e}{3}\left(B_{Z}^{2}\right)_{Z}+\frac{\sqrt{6} R e^{2}}{3}\left(B_{Z}^{3}\right)_{Z} .
$$

When expressed in terms of a new variable $\phi=B_{Z}$, this expression has the form of the Cahn-Hilliard equation (the term, $B_{Z}^{2}$, can be eliminated by setting $B_{Z}=\phi+C$, where $C$ is a suitable constant, placing the system in the usual Cahn-Hilliard form).

Note that the nonlinearity is cubic in (3.11). This resulted entirely because the coefficient of the otherwise leading quadratic term $\left(B_{Z}^{2}\right)_{Z}$ is proportional to $(G-2)$, 
and we successfully pushed that term to the same order as the cubic nonlinearity by making the codimension-two parameter choices in (3.3). Quadratic nonlinear terms cannot be discounted by symmetry arguments in the current expansion because the governing system has the reflection symmetry transformations, $(x, \psi) \rightarrow(-x,-\psi)$ and $(x, z, b) \rightarrow(-x,-z,-b)$. From these symmetries alone, we see that the amplitude cannot be the standard Cahn-Hilliard equation, because that equation is invariant under the independent transformations, $B \rightarrow-B$ and $Z \rightarrow-Z$.

A Lyapunov functional exists for the Cahn-Hilliard equation which predicts that the evolution of the system is the inexorable convergence to the steady solution with the largest spatial scale (e.g. Chapman \& Proctor 1980). The convergence, however, can be delayed for long periods by meta-stable states consisting of a sequence of layers separated by slowly drifting interfaces. (An illustration of the coarsening process is given below.) It is this property of the Cahn-Hilliard model that leads us to predict that layering can result in laminar flows.

\subsection{Weak viscous instability}

The marginal stability condition for viscous instability, viewed as a critical Reynolds number, $R e=R e_{c}$, is

$$
R e_{c}^{2}=\frac{2(1+G)^{3}}{1-\sigma G},
$$

which also fixes the Péclet number given the Prandtl number. To push the system into a weakly unstable regime, we set $R e=R e_{c}+\epsilon^{2} R e_{2}$, and again perform an asymptotic expansion. We begin with the sequences,

$$
\psi=\psi_{0}+\epsilon \psi_{1}+\cdots, \quad b=b_{0}+\epsilon b_{1}+\cdots,
$$

and select a leading-order solution,

$$
\psi_{0}=A(Z, \tau)+\frac{1}{1+G} \cos x, \quad b_{0}=\frac{G}{\operatorname{Re}(1+G)} \sin x,
$$

which, this time, contains only the slow viscous mode.

At order $\epsilon^{2}$, we find the relations,

$$
\begin{gathered}
\psi_{1 x x x x}+\operatorname{Re}_{c} b_{1 x}=\operatorname{Re}_{c}\left(\psi_{0 x} \psi_{0 x x Z}-\psi_{0 Z} \psi_{0 x x x}\right), \\
G \psi_{1 x}-\operatorname{Re}_{c} b_{1 x x}=\sigma \operatorname{Re}_{c}^{2}\left(b_{0 x} \psi_{0 Z}-b_{0 Z} \psi_{0 x}\right),
\end{gathered}
$$

which are solved by

$$
\psi_{1}=\frac{\operatorname{Re}(\sigma G-1)}{(1+G)^{2}} A_{Z} \sin x, \quad b_{1}=B_{1}(Z, \tau)+\frac{G(1+\sigma)}{(1+G)^{2}} A_{Z} \cos x .
$$

In (3.17), we add a mean buoyancy term; it turns out that this is necessary because the slow viscous mode forces a mean response in $b$ at order $\epsilon$, as is clear from the vertical average of (3.2), which provides the relation,

$$
\sigma \operatorname{Re}_{c} \overline{\left(\psi_{1 x} b_{1 z}-\psi_{1 z} b_{1 x}-\psi_{2 z} b_{0 x}+\psi_{0 x} b_{2 Z}-\psi_{0 z} b_{2 x}\right)}=\overline{b_{1 Z Z}} \equiv B_{1 Z z}
$$

at order $\epsilon^{3}$. The left-hand side does not, in general, vanish, thus forcing $B_{1}$. We delay the construction of this quantity until we solve the system at next order. We again place some of the details in the Appendix, and arrive at the relation,

$$
B_{1 Z Z}=\frac{\sigma G\left(1-2 G \sigma+\sigma^{2}\right)\left(A_{Z}^{2}\right)_{Z}}{(1+G)\left(1+\sigma^{2}-\sigma G-\sigma^{2} G\right)} \equiv \Gamma\left(A_{Z}^{2}\right)_{Z} .
$$


At this stage we must make some statement about boundary conditions in $Z$; we adopt periodicity in this direction. The integral of (3.19) then implies that

$$
B_{1 Z}=\Gamma\left(A_{Z}^{2}-\left\langle A_{Z}^{2}\right\rangle\right)
$$

where the angular brackets denote the average in $Z$.

Finally, we proceed to third order and evaluate the vertical average of (3.1). This leads to the amplitude equation:

$$
\begin{aligned}
A_{\tau}= & {\left[\frac{\operatorname{Re}_{c}^{3} \sigma(5-2 \sigma G+3 \sigma)}{6(1+G)^{4}} \Gamma\left\langle A_{Z}^{2}\right\rangle-\frac{2 R e_{2}}{R e_{c}}\right] A_{Z Z} } \\
& -\frac{3 R e_{c}}{2(1+G)^{5}}\left[1-\sigma G+\frac{\sigma^{2} R e_{c}^{2} G(\sigma G-8 \sigma-3)}{24(G+1)(G+16)}\right] A_{Z Z Z Z} \\
& +\frac{R e_{c}^{3}}{6(1+G)^{4}}\left[\sigma \Gamma(5-2 \sigma G+3 \sigma)+\frac{2-6 \sigma G+\sigma^{2} G(G-\sigma G-3 \sigma-5)}{1+G}\right]\left(A_{Z}^{3}\right)_{Z} .
\end{aligned}
$$

If $G=0$, this equation reduces to a Cahn-Hilliard equation for the variable, $A_{Z}$, and is equivalent to a system derived previously by Sivashinksy (1985). However, with $G \neq 0$, it is not precisely of Cahn-Hilliard form because of the non-local term involving $\overline{A_{Z}^{2}}$.

The negative diffusion term in (3.21) amplifies gradients of $A_{Z}$, and therefore $B_{Z}$. These gradients continue to sharpen as the instability operates, but can saturate when the nonlinear diffusion term comes into play. In the standard Cahn-Hilliard system, such a saturation is guaranteed if the coefficient in front of the cubic nonlinearity, $\left(A_{Z}^{3}\right)_{Z}$, is positive. For our model in (3.21), the cubic coefficient is indeed positive when the stratification parameter, $G$, is small. However, as $G$ increases, the cubic coefficient decreases and can eventually change sign. In this circumstance, one anticipates that the leading nonlinearity cannot saturate the sharpening of the interfaces, but accelerates it until further nonlinear terms become important. This situation is analogous to a subcritical bifurcation (a connection that can be made firmer by decomposing $A$ into normal modes in $Z$ and performing a standard amplitude expansion). In other words, by stratifying the fluid, we can force the viscous instability to become subcritical, creating a 'harder' transition at onset. Also, with the non-local term, $\left\langle A_{Z}^{2}\right\rangle$, it is not clear what remains of the coarsening dynamics described by the Cahn-Hilliard equation. We solve a more general version of the amplitude equation (3.21) below to shed some light on this second issue.

\subsection{Long-wave equations by projection}

An alternative approach to the asymptotic expansion above is provided by a Galerkin projection of the form,

$$
\begin{aligned}
& \psi=A(z, t)+\sum_{j=1}^{3}\left[a_{1, j}(z, t) \cos j x+a_{2, j}(z, t) \sin j x\right], \\
& b=B(z, t)+\sum_{j=1}^{3}\left[b_{1, j}(z, t) \cos j x+b_{2, j}(z, t) \sin j x\right],
\end{aligned}
$$

where the coefficients $a_{1, j}, a_{2, j}, b_{1, j}$ and $b_{2, j}$ can be determined in terms of $A$ and $B$ by introducing the projection into the governing equations with the time derivatives neglected. Substitution of the projection into the horizontal averages of the governing 
equations then provides the evolution equations for $A$ and $B$. A mammoth amount of algebra, greatly assisted by MAPLE, eventually yields the system,

$$
\begin{aligned}
A_{t}= & R e^{-1}\left[1-\frac{R e^{2}(1-\sigma G)}{2(1+G)^{3}}\right] A_{z z}+\frac{\sigma R e^{3}}{2(1+G)^{4}}\left[2(1-\sigma G) A_{z z} B_{z}+(1+\sigma)\left(A_{z} B_{z}\right)_{z}\right] \\
& -\frac{3 R e}{2(1+G)^{4}}\left[1-\sigma G+\frac{\left.\sigma^{2} R e^{2} G(\sigma G-8 \sigma-3)\right]}{24(G+16)(G+1)}\right] A_{z z z z} \\
& -\frac{3 \sigma^{2} R e^{5}}{2(1+G)^{5}}(2-\sigma G+\sigma) B_{z}^{2} A_{z z}-\frac{2 \sigma^{2} R e^{5}}{(1+G)^{5}}(1+\sigma) A_{z} B_{z} B_{z z} \\
& +\frac{R e^{3}}{6(1+G)^{5}}\left[2-6 \sigma G+\sigma^{2} G^{2}(1-\sigma)-\sigma^{2} G(3 \sigma+5)\right]\left(A_{z}^{3}\right)_{z} \\
B_{t}= & P e^{-1}\left[1-\frac{\sigma^{2} R e^{2}(G-1)}{2(1+G)^{3}}\right] B_{z z}+\frac{R e}{2(1+G)^{4}}\left[G\left(2 G \sigma-\sigma^{2}-1\right) A_{z}^{2}\right. \\
& \left.+\sigma^{2} \operatorname{Re}^{2}(G-2) B_{z}^{2}\right]_{z}-\frac{\sigma R e}{2(1+G)^{4}}\left[5 G-1+\frac{\sigma R e^{2}\left(9 \sigma G-8 \sigma+6 G-\sigma G^{2}\right)}{8(G+16)(G+1)}\right] B_{z z z z} \\
& +\frac{\sigma^{3} R e^{5}(3-G)}{2(1+G)^{5}}\left(B_{z}^{3}\right)_{z}+\frac{\sigma R e^{3}}{2(1+G)^{5}}\left[(3 G-1)\left(\sigma^{2}+1\right)+4 \sigma G(1-G)\right]\left(A_{z}^{2} B_{z}\right)_{z} .
\end{aligned}
$$

This system displays all the symmetries of the original equations and reduces to the asymptotic models above in suitable limits of the parameters. However, it is not necessary to restrict the parameter settings to those values. Moreover, the system captures the dynamics of the situation in which both the viscous and conductive instabilities become unstable at the same time, taking the same form as equations derivable by asymptotic means. Hence, (3.23)-(3.24) provide a more compact description of the dynamics, and we solve this system numerically to gain further insight into the nonlinear behaviour. Note that the growth of $A$ stimulates $B$, but $B$ evolves on its own if $A(z, 0)=0$.

We illustrate the weakly nonlinear dynamics captured by the model in figures 4-6. Figure 4 shows a computation in which the conductive instability arises, but there is no viscous instability. In this case, $A$ decays to zero, and $B_{Z}$ forms a sequence of alternating layers (five layers with positive and five with negative gradient appear initially, creating the pattern of stripes in the figure). As time proceeds, the interfaces bordering the layers drift under mutual interaction. At various instants, interfaces approach one another and collide, thereby removing one of the layers, and widening its neighbours. As a result of this process, the characteristic lengthscale of the pattern gradually increases with time; this is the coarsening dynamics captured by CahnHilliard (to which the system reduces when $A \rightarrow 0$ ). At the end of the simulation, only four interfaces remain (two pairs of layers). Another collision occurs at later times (not shown) to coarsen the pattern to its ultimate final state, a pattern with the longest spatial scale. In other words, the pure conductive mode exhibits a completed inverse cascade. Associated with this cascade are rearrangements of the stratification that take the form of a staircase in the full buoyancy distribution. Also, even though the horizontal average of the velocity field decays, the motions driven by the conductive mode do not. The Fourier-series form of the solution suggests slowly evolving cellular patterns in the velocity field, as observed in the computations described in the next section. 

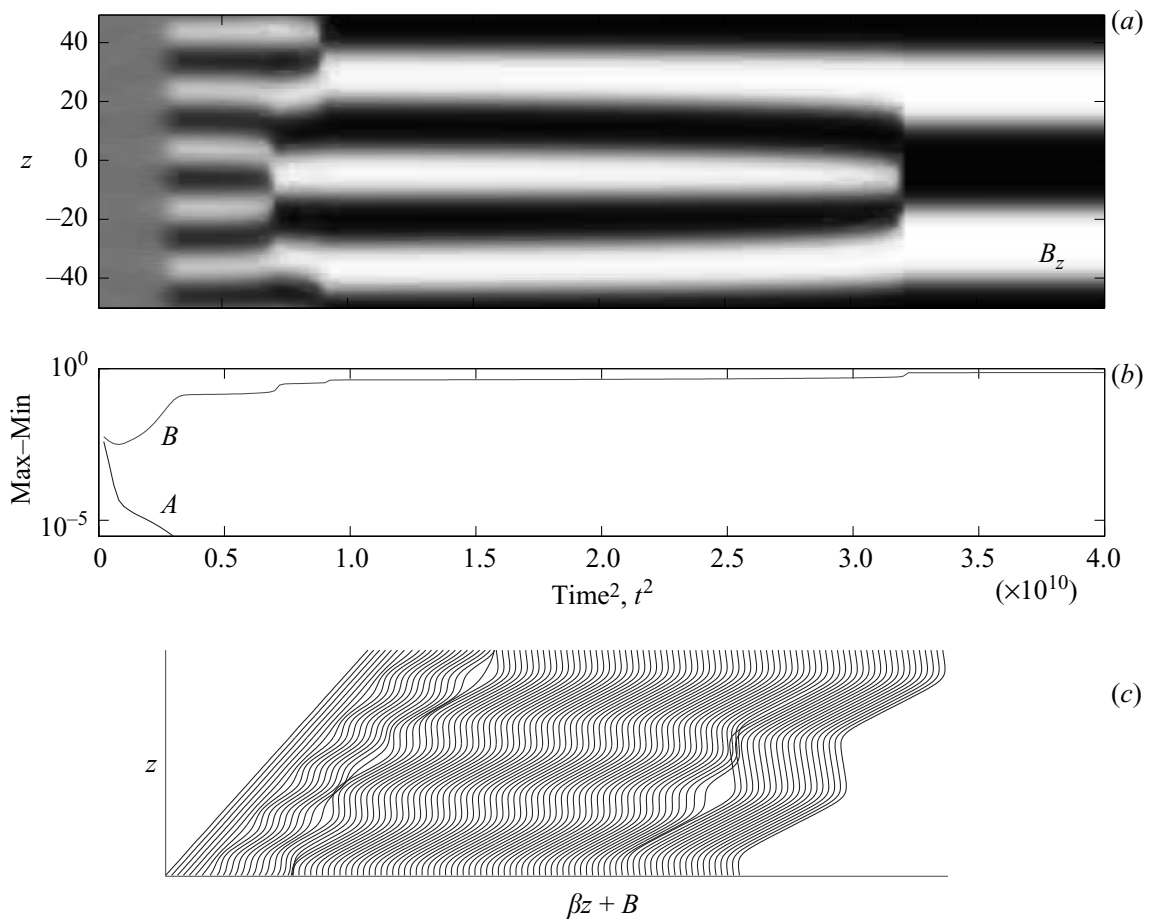

(c)

Figure 4. Conductive instability in a domain of size 100. (a) A greyscale of $B_{z}$ on the $\left(t^{2}, z\right)$ plane. (b) The time history of the peak-to-peak amplitudes of $A$ and $B$. (c) The evolving, total buoyancy field, $\beta z+b$, as a sequence of snapshots successively offset to the right. The parameter values: $R e=6, G=2$ and $\sigma=2$. In this computation, the sign of the coefficient of the term $A_{z z z z}$ in (3.23) has been reversed to ensure that the system remains well-posed. The significance of positive sign of this coefficient is that short waves are predicted to be unstable (an ultra-violet catastrophe), which is unphysical and probably an artifact of the long-wave expansion. Although the switch of sign is a little arbitrary, it has the same effect as including a regularizing term with higher derivatives provided there are no instabilities with short wavelengths.

Figure 5 displays the emergence of the (supercritical) viscous instability in the absence of a conductive one. When the viscous instability first enters the nonlinear regime, a pattern with five light and dark stripes appears in $A_{z}$. These stripes reflect an alternating sequence of horizontally directed jets superposed on the underlying vertical flow (which corresponds to a characteristic meandering motion that is visible in the numerical solutions of the next section). Sharp negative buoyancy gradients build up in the shear layers bordering the jets, giving a characteristic vertical scale to $b$ which is twice that of the velocity field. However, the effect on the total buoyancy field is less pronounced and little forms by way of a staircase. Note that the interface collapses in figure 5 generate a response throughout the entire pattern (the overall shading of the layers and interfaces appears to abruptly change at the collisions, especially for $B(z, t))$, in contrast to the relatively local effects seen in figure 4 . This reflects a more non-local nature of the dynamics which is also expected from the non-local term in (3.21). Moreover, the pattern does not coarsen further at later times, unlike in figure 4, and the state ending figure 5 appears to be the final one. Thus coarsening is arrested, as found in our earlier paper for much weaker stratification. 

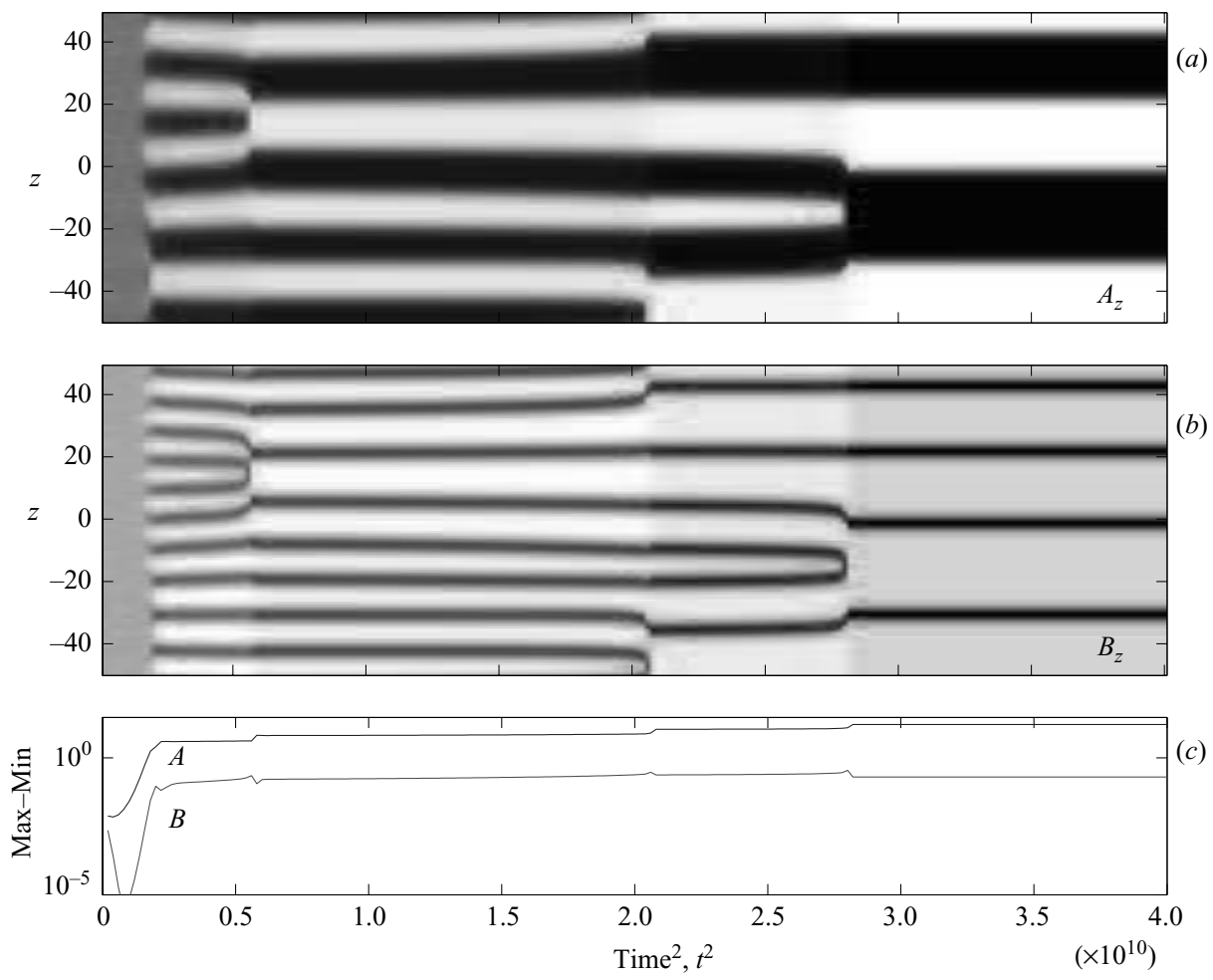

(d)
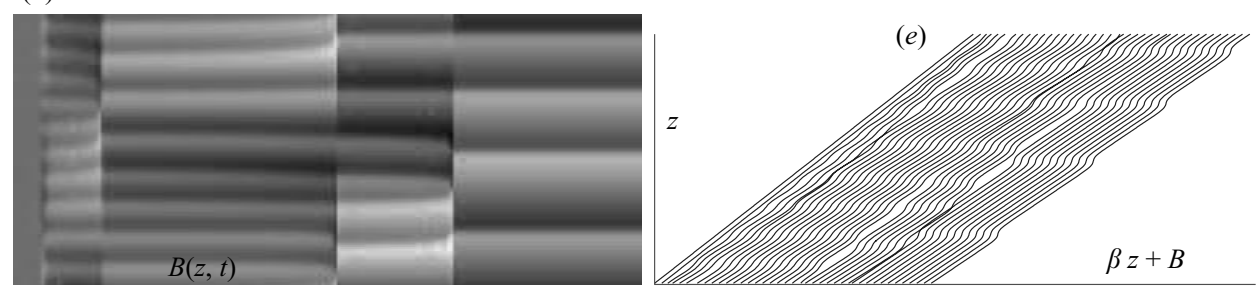

FIGURE 5. Viscous instability in a domain of size 100: $(a, b)$ greyscales of $A_{z}$ and $B_{z}$ on the $(t, z)$-plane; $(c)$ time series of the peak-to-peak amplitudes of $A$ and $B ;(d)$ a greyscale of $B$ on the $(t, z)$-plane; and $(e)$ a sequence of snapshots of total buoyancy, $\beta z+b$. The parameter values: $R e=3, G=1 / 4$ and $\sigma=1 / 2$.

Figure 6 shows a computation in which both modes are unstable and a steady, noncoarsening pattern emerges. This picture illustrates another feature of the dynamics, namely that when the two modes compete, the viscous mode dominates and suppresses the conductive mode. In order to emphasize this feature of the dynamics in the computation, the growth rate of the conductive mode was artificially increased and the initial condition seeded with the unstable conductive mode in order to promote that instability over the viscous mode, at least initially. After a period of time, however, the viscous mode overtakes the conductive mode and establishes a steady pattern that shows no coarsening.

We close this section by cautioning that the long-wave model in (3.23)-(3.24) is not always well-posed: for certain parameter choices, the hyper-diffusion terms, $A_{z z z z}$ and 

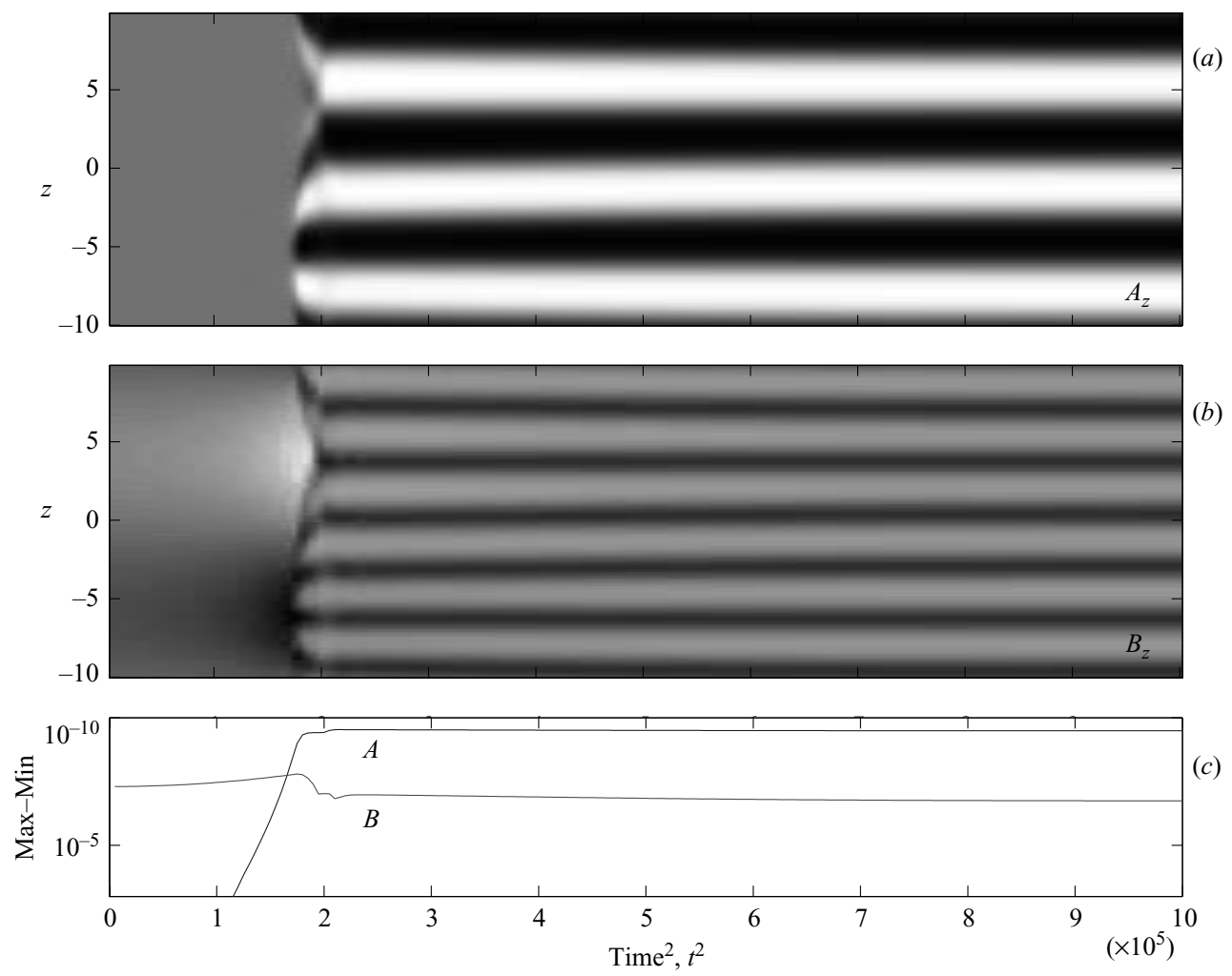

FiguRE 6. Double instability in a domain of size 20. $(a, b)$ Greyscales of $A_{z}$ and $B_{z}$ on the $(t, z)$-plane, and (c) the peak-to-peak amplitudes of $A$ and $B$. The parameter values: $R e=75$, $G=2.5$ and $\sigma=0.11$. In order to promote artificially the conductive mode over the viscous mode, the growth rate of the conductive instability has been increased unphysically by a factor of 20 , and the initial condition seeded mainly with the unstable linear conductive mode.

$B_{z z z z}$, turn out to have positive coefficients. (In figure 4, for example, even though the $A$-mode decayed away, we artificially reversed the sign of the $A_{z z z z}$ term to ensure the system remained well-posed.) Moreover, the coefficients of the nonlinear terms, $\left(B_{z}\right)^{3}$ and $\left(A_{z}\right)^{3}$, are not always positive and the system seems able to pass abruptly into a phase where gradients are continually sharpened and the computation breaks down. Of course, since the original system is unlikely to be prone to the same problems, the fault must lie in the Galerkin truncation. We avoid any such problems below by solving the full governing equations numerically.

\section{Direct numerical simulations}

To compare the results of the previous section with numerical solutions we select three sets of representative parameter values:

(i) Conductive: $\operatorname{Re}=6, G=2, \sigma=2(P e=12, \beta=1 / 36, \theta=0)$,

(ii) Viscous: $R e=3, G=1 / 4, \sigma=1 / 2(P e=3, \beta=1 / 18, \theta=0)$,

(iii) Combination: $\operatorname{Re}=17, G=1.705, \sigma=1 / 2(P e=8.5, \beta=1 / 85, \theta=0)$, 
(a)

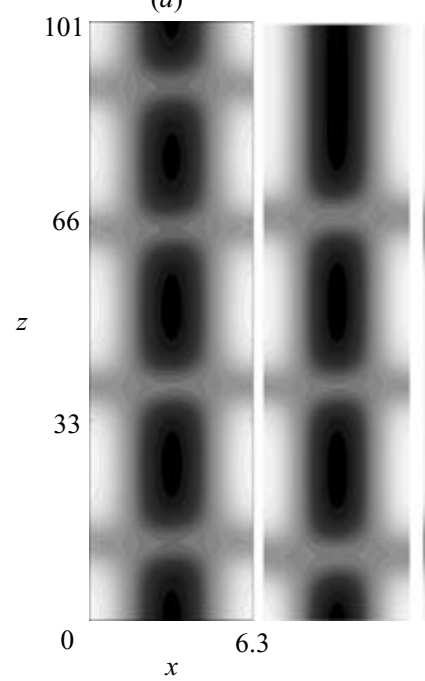

(b)

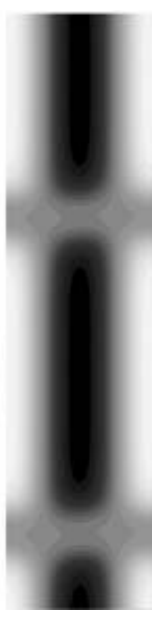

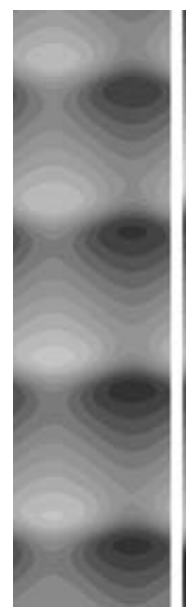
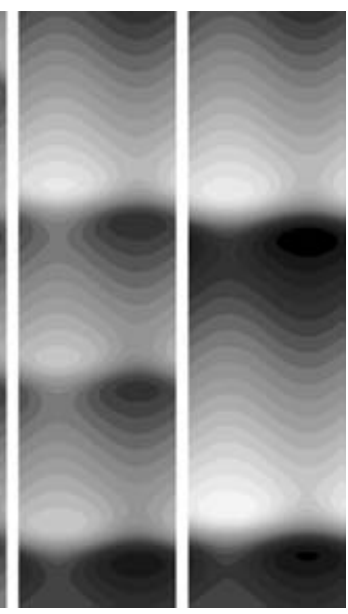

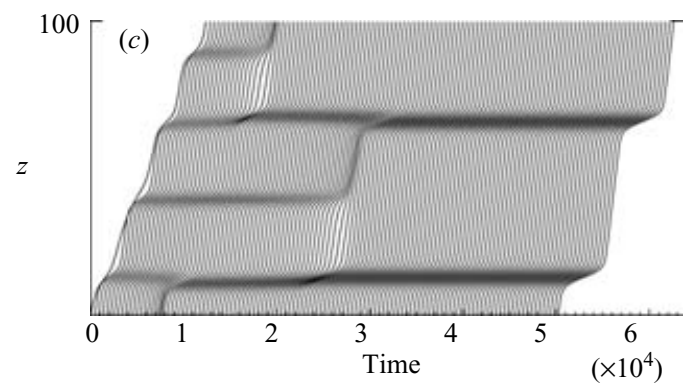

FIgURE 7. Conductive instability in the domain $[2 \pi, 32 \pi]$. Snapshots of $(a) \psi(x, z, t)$ and (b) $b(x, z, t)$ as greyscales on the $(x, z)$-plane. The parameter values: $R e=6, G=2$ and $\sigma=2$. The times of the snapshots are 5000, 15000 and 26000 (left to right). (c) The evolution of the total, horizontally averaged buoyancy field, $\beta z+B(z, t)$.

with a domain of horizontal and vertical lengths, $L_{x}=2 n \pi$ and $L_{z}=32 \pi$. We explore cases with $n=1$ or 2 : if $n=1$, there is a single pair of opposed vertical jets in the domain; for $n=2$, there are two pairs.

Figure 7 shows the evolution of case (i), with a single pair of jets. The instability creates a cellular velocity pattern that is also characterized by a 'blobby' field of buoyancy anomaly. There is weak mean ( $x$-averaged) horizontal flow, but strong rearrangement of the mean ( $x$-averaged) buoyancy into a staircase, both of which are typical of the conductive instability. The pattern initially forms an array of four cellular structures, but these coarsen to two, as expected from the underlying CahnHilliard dynamics.

Figure 8 shows the evolution of a single jet pair in case (ii). The instability initiates a distinctive meander of the jet and eddies emerge within parts of the meander; this pattern is much like that arising in weakly stratified Kolmogorov flow consider in our earlier paper (although there the underlying jets were horizontally directed). The meandering reflects stronger mean horizontal flow, and the buoyancy field shows twice the vertical scale of the horizontal field. The eddying meanders coarsen from six to four, with large transients produced in the buoyancy field as a result of the 
(a)

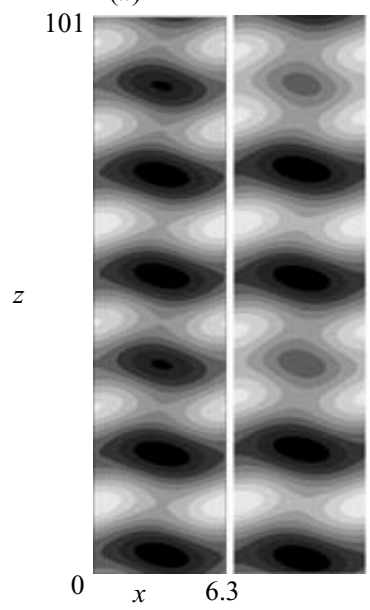

(c)

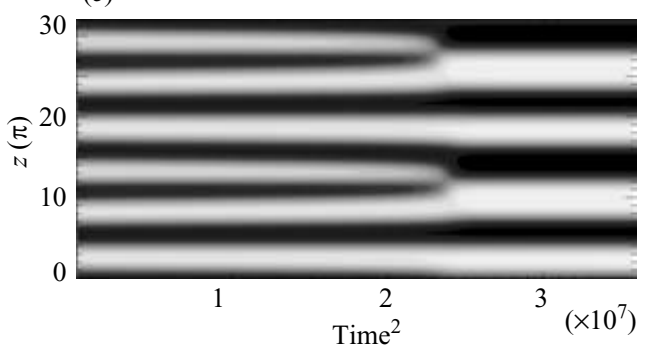

(b)

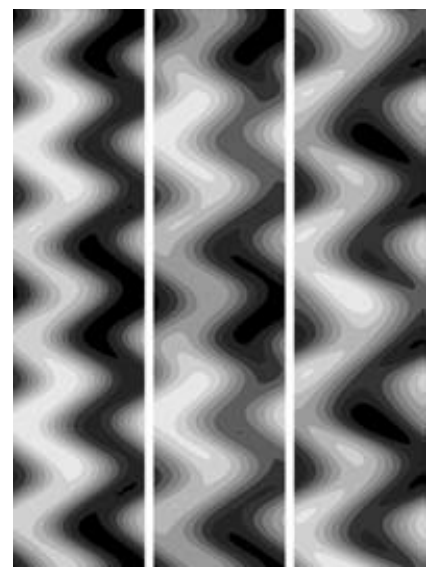

$(d)$

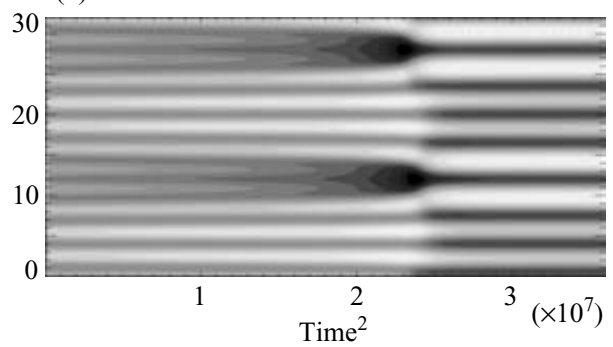

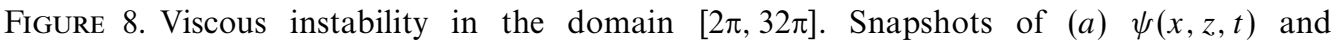
(b) $b(x, z, t)$ as greyscales on the $(x, z)$-plane. The horizontal averages of $(c) \psi$ and $(d) b$ on the $(t, z)$-plane. The parameter values: $R e=3, G=1 / 4$ and $\sigma=1 / 2$. The times of the snapshots are $1.5 \times 10^{3}, 4.6 \times 10^{3}$, and $6 \times 10^{3}$, respectively (from left to right).

mergers. At four meanders, coarsening appears to halt. Again, these features reflect the weakly nonlinear dynamics predicted by the long-wave theory.

The single pair of jets for case (iii) is shown in figure 9. The forming patterns bear much in common with those created by the viscous instability, although there is little sign of coarsening in the meanders. As expected the conductive mode is suppressed.

Results for $n=2$ are shown in figures 10 and 11. The main surprise for case (i) is that the layering pattern of the conductive mode becomes replaced by a pattern with the meandering and eddying character of the viscous mode. The pathway to this final state proceeds in two stages. First, a pattern emerges with the blobby and cellular features typical of the conductive mode, but it is unsteady, irregular and contains a significant contribution from a subharmonic, $x$-wavenumber-one disturbance. This initial phase is interrupted by the growth of what appears to be a secondary instability that spawns the meandering and eddying jet pattern. Case (ii) shows no surprises, being a periodically repeated version of the $n=1$ single paired jets, and is not therefore displayed. Meandering and eddying again predominate in the case (iii) computation, but now, somewhat surprisingly, significant coarsening takes place. This reduces the initial number of meanders from seven down to three. From the trend of the computation, we suspect that a further coarsening to two would take place around $t=2 \times 10^{4}$ if we were to continue the computation. The coarsening is now mediated by an oscillatory interaction involving the horizontal motion of buoyancy anomalies 
(a)

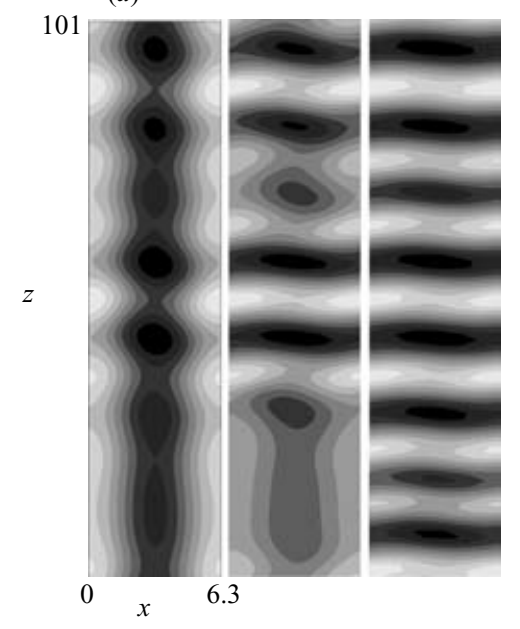

(b)

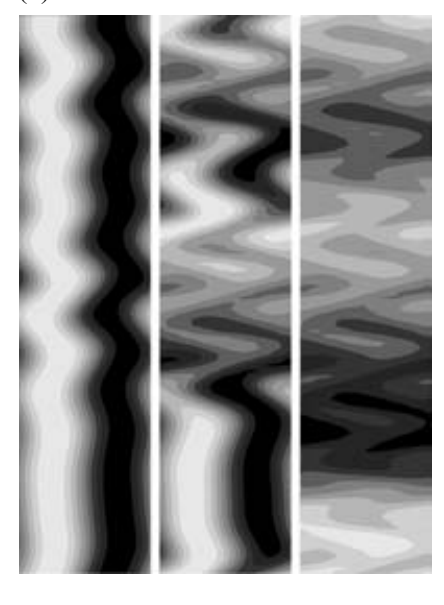

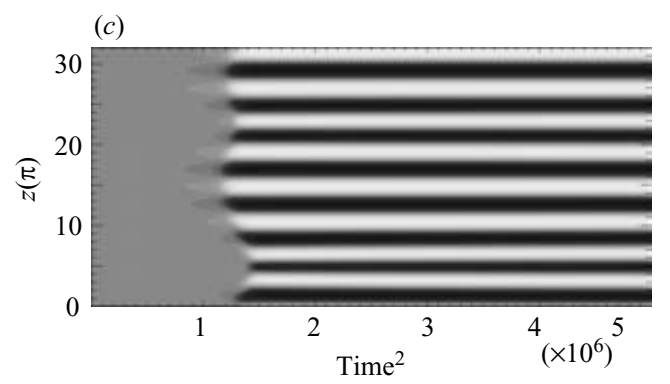

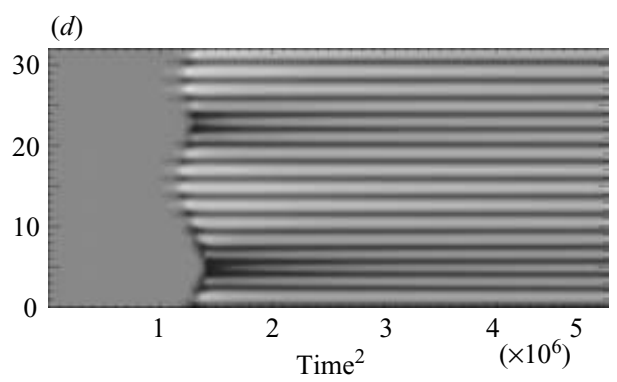

FIGURE 9. Double instability in the domain $[2 \pi, 32 \pi]$. The final distribution (at $t=1219$ ) of (a) $\psi(x, z, t)$ and $(b) b(x, z, t)$ as greyscales on the $(x, z)$-plane. The horizontal averages of $(c) \psi$ and $(d) b$ on the $(t, z)$-plane. The parameter values: $R e=17, G=1.705$ and $\sigma=0.5$. The times of the snapshots are $1.04 \times 10^{3}, 1.12 \times 10^{3}$, and $1.22 \times 10^{3}$, respectively (from left to right).

and pulsations of the eddies. The plots of horizontally averaged streamfunction and buoyancy anomaly nicely bring out this feature, and also illustrate how strong layer migrations occur in this computation.

Finally, we remark that we have also run some further computations at either higher Reynolds numbers, or with inclined jets. At higher Reynolds number, little visibly remains of the structured patterns seen just above onset, which are replaced by complicated time-dependent motions, at least for small $G$. Computations for larger $G$ and Reynolds number showed steady states with a strong viscous mode and weak layering, even under conditions where the viscous mode was expected to be relatively strongly damped. We attribute this dynamics to the viscous mode becoming subcritical and growing nonlinearly. Simulations with inclined jets reveal a different dynamics still, involving wavy, time-dependent patterns, but we end the exploration here.

\section{Concluding remarks}

We have explored instability of forced stratified shear flow. The picture is largely one of two modes of instability that develop over long vertical scales. The first is a 'viscous' instability which is related to the classical instability of Kolmogorov flow. The introduction of stratification can suppress this instability, but can also 
(a)

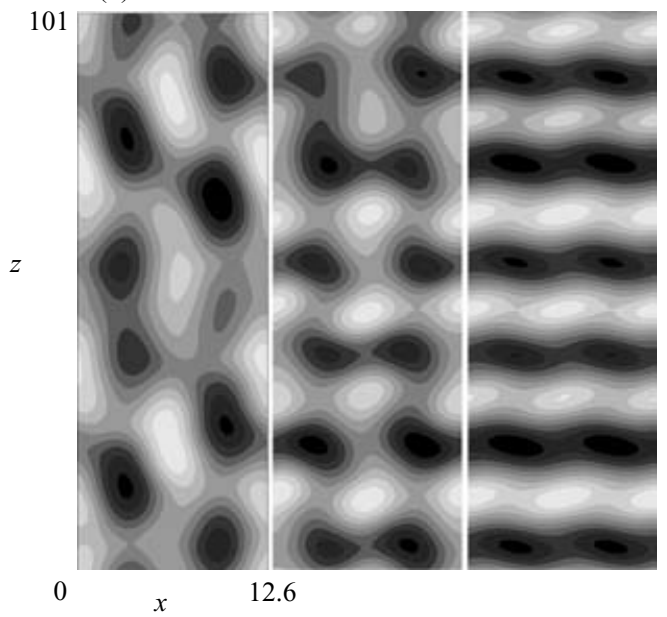

(c)

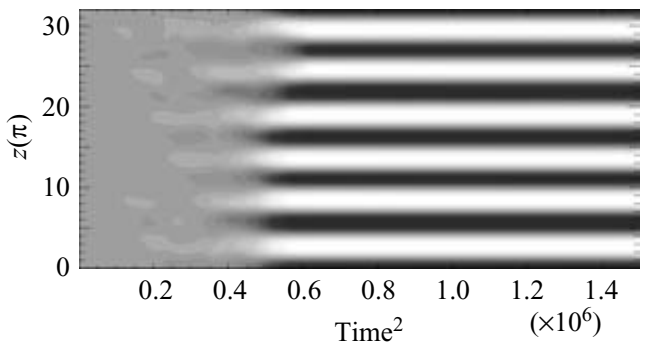

(b)

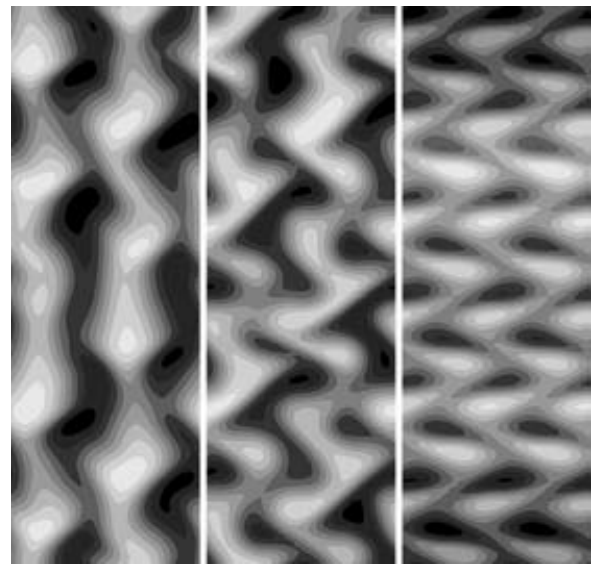

(d)

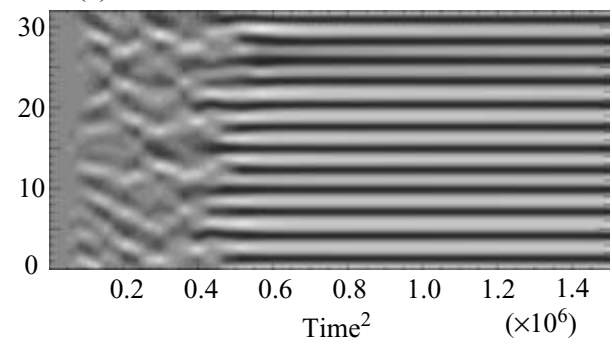

FIGURE 10. Conductivity instability with two paired jets. $(a-d)$ As figure 9 . The times of the snapshots are 390,670 , and $1.44 \times 10^{3}$, respectively (from left to right).

make it subcritical. The second instability is a 'conductive one' which operates by creating a large-scale negative thermal diffusion. This instability arises with stronger stratification and creates prominent staircases in the buoyancy field. The steps of the staircase have their own nonlinear dynamics, and often show 'coarsening' - the merger of steps as the dividing interfaces collide, with the subsequent lengthening of the scale of the overall pattern. The viscous instability appears able to dominate and suppress the conductive mode should both operate, and creates little by way of layering in the density field. Moreover, there is a tendency for that mode to become subcritical and dominate by growing nonlinearly, even under conditions where it is linearly stable. Thus, although it seems possible that the conductive instability is the laminar precursor of the fluid phenomenon that generates turbulent staircases, it is essential to remove the viscous mode. In our previous investigation (Balmforth \& Young 2002), the configuration and parameter range are such that only viscous instability is present, and thus almost no evidence of layering is observed in that case.

Instabilities catalyzed by viscosity or thermal conduction are known in a variety of other contexts, notably differentially rotating annular columns (Yih 1961) and stellar interiors (Goldreich \& Schubert 1967), and baroclinic vortices (McIntyre 1970). One is tempted to rationalize the current instabilities as analogies of those examples. However, there are differences in the current theory. For example, a general conclusion reached in these other problems is that conductive instability is typical of 
(a)

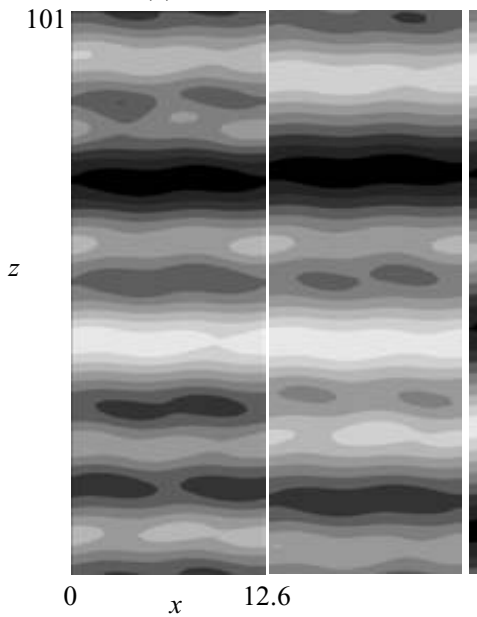

(c)

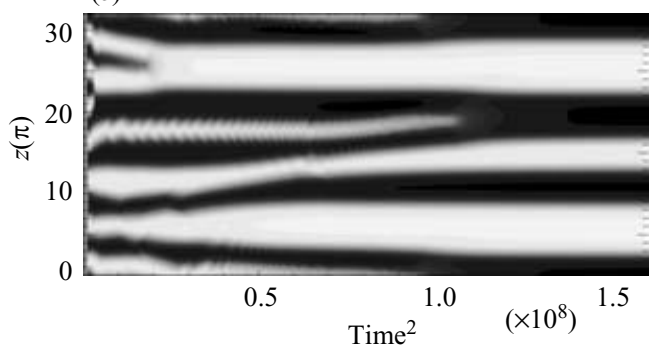

(b)
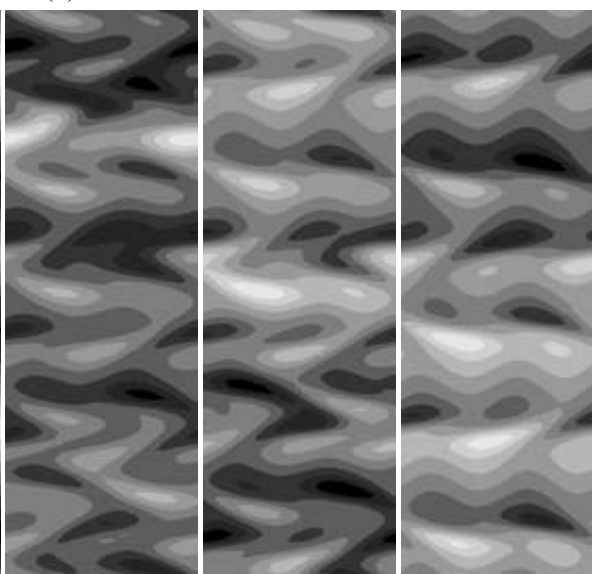

(d)

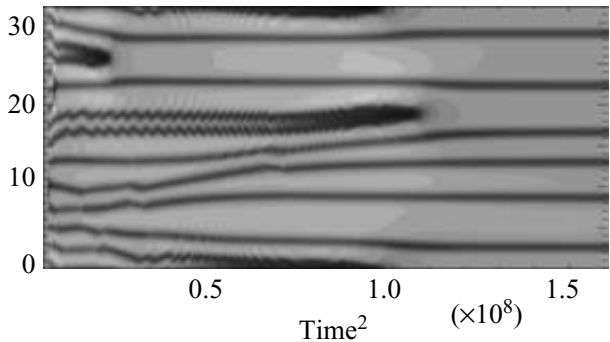

Figure 11. Combined instability with two paired jets. $(a-d)$ As figure 9 . The times of the snapshots are 1080, 1890, and $1.432 \times 10^{4}$, respectively (from left to right).

low Prandtl number $(\sigma<1)$, and viscous instability of high Prandtl number $(\sigma>1)$, which is not found here.

This work was supported by the National Science Foundation (Collaborations in Mathematical Geosciences, grant ATM0222109). We thank W. R. Young for supplying many of the ideas that this work was founded upon, and for many useful discussions. Y.-N. Y. acknowledges support from CTR.

\section{Appendix. Formulae for weakly nonlinear theory}

\section{A.1. Weak conductive instability}

The order $\epsilon^{3}$ solution is

$$
\begin{aligned}
& \psi_{3}= \frac{13 R e}{72}\left(\frac{G_{1}}{3}+R e \sqrt{6} B_{Z}\right) B_{Z Z} \sin 2 x+\frac{R e}{5976}(10 \sqrt{6}+3 R e) B_{Z Z Z} \cos 3 x \\
&- {\left[\frac{1}{3}\left(\frac{G_{1}}{3}+\sqrt{6} \operatorname{Re} B_{z}\right)^{3}+\frac{R e}{72}(R e+30 \sqrt{6}) B_{Z Z Z}+\frac{\sqrt{6}}{9} \kappa_{2}\left(\frac{G_{1}}{3}+\sqrt{6} \operatorname{Re} B_{Z}\right)\right] \cos x, } \\
& b_{3}=\frac{13}{9}\left(\frac{G_{1}}{3}+\operatorname{Re} \sqrt{6} B_{Z}\right) B_{Z Z} \cos 2 x-\frac{1}{2988}(135 \sqrt{6}-\operatorname{Re}) B_{Z Z Z} \sin 3 x \\
&-\left[\left(\frac{R e}{36}+\sqrt{\frac{3}{8}}\right) B_{Z Z Z}-\frac{1}{3 R e}\left(\frac{G_{1}}{3}+\sqrt{6} \operatorname{Re} B_{Z}\right)^{3}-\frac{\sqrt{6}}{9 R e} \kappa_{2} G_{1}-2 \kappa_{2} B_{Z}\right] \sin x .
\end{aligned}
$$




\section{A.2. Weak viscous instability}

At order $\epsilon^{2}$, we find

$$
\begin{gathered}
\psi_{2 x x x x}+\operatorname{Re}_{c} b_{2 x}=\operatorname{Re}_{c}\left(\psi_{0 x} \psi_{1 x x z}+\psi_{1 x} \psi_{0 x x z}-\psi_{1 Z} \psi_{0 x x x}-\psi_{0 z} \psi_{1 x x x}\right)-2 \psi_{0 x x Z Z}, \\
G \psi_{2 x}-\operatorname{Re}_{c} b_{2 x x}=\sigma R e_{c}^{2}\left(b_{1 x} \psi_{0 Z}+b_{0 x} \psi_{1 Z}-b_{0 Z} \psi_{1 x}-b_{1 Z} \psi_{0 x}\right)+R e_{c} b_{0 Z Z},
\end{gathered}
$$

which gives

$$
\begin{aligned}
& \psi_{2}=\left[\frac{2 \sigma G-1+\sigma^{2} G}{1+G} A_{Z}^{2}-\sigma B_{1 Z}\right] \frac{R e_{c}^{2} \cos x}{(G+1)^{2}}-\frac{\sigma^{2} G R e_{c}^{2} A_{Z Z} \cos 2 x}{4(1+G)^{2}(16+G)}, \\
& b_{2}=\left[\sigma B_{1 Z}-\frac{G \operatorname{Re}_{c}\left(1-\sigma G+\sigma+\sigma^{2}\right)}{(G+1)} A_{Z}^{2}\right] \frac{\sin x}{(1+G)^{2}}+\frac{2 \sigma^{2} \operatorname{Re}_{c} G A_{Z Z} \sin 2 x}{(1+G)^{2}(G+16)} .
\end{aligned}
$$

At order $\epsilon^{3}$ :

$$
\begin{gathered}
\psi_{3}=\frac{Q_{1} R e_{c} \sin x}{2(1+G)^{4}}+\left[\frac{10 G(G+4)(\sigma+1)}{(1+G)(16+G)} A_{Z} A_{Z Z}-B_{1 Z Z}\right] \frac{\sigma^{2} R e_{c}^{3} \sin 2 x}{8(G+16)(1+G)^{2}} \\
\left.-\frac{\sigma^{2} G R e_{c}^{3}(9+\sigma G+8 \sigma)}{48(81+G)(G+16)(1+G)^{3}} A_{Z Z Z} \sin 3 x, \quad \text { (A } 5\right) \\
b_{3}=\frac{Q_{2} \cos x}{(1+G)^{4}}-\left\{\frac{G\left[G^{2}-3 G-64-20(4+\sigma)\right]}{2(G+16)(1+G)} A_{Z} A_{Z Z}+B_{1 Z Z}\right\} \frac{\sigma^{2} R e_{c}^{2} \cos 2 x}{(G+16)(1+G)^{2}} \\
+\frac{\sigma^{2} G R e^{2}(G-9 \sigma G-72 \sigma)}{8(81+G)(G+16)(1+G)^{3}} A_{Z Z Z} \cos 3 x
\end{gathered}
$$

with

$$
\begin{gathered}
Q_{1}=\sigma \operatorname{Re}_{c}^{2}(1+G)(2-\sigma G+\sigma) A_{Z} B_{1 Z Z}+\left[\frac{G \sigma^{2}(8 \sigma-\sigma G+3)}{8(16+G)}-3(1+G)(1-\sigma G)\right] A_{Z Z Z} \\
+\operatorname{Re}_{c}^{2}\left(\sigma^{2} G^{2}-\sigma^{3} G-2 \sigma^{2} G-3 \sigma G+1\right) A_{Z}^{3} \quad(\mathrm{~A} 7) \\
Q_{2}= \\
+\left[(1+G)(4-2 \sigma G+G+\sigma)-\frac{\sigma^{2} e_{c}^{2}\left(1-G_{c}^{2}(\sigma G-8 \sigma+3 G)\right.}{8(G+16)}\right] G A_{Z Z Z} \\
+[\text { (A } 8)
\end{gathered}
$$

\section{REFERENCES}

Balmforth, N. J., Llewellyn Smith, S. G. \& Young, W. R. 1998 Dynamics of steps and layers in a turbulent stratified fluid. J. Fluid Mech. 400, 500-500.

Balmforth, N. J. \& Young, Y.-N. 2002 Stratified Kolmogorov flow. J. Fluid Mech. 450, 131-167.

Chapman, C. \& Proctor, M. 1980 Nonlinear Rayleigh-Bénard convection with poorly conducting boundaries. J. Fluid Mech. 101, 759-782.

Drazin, P. G. 1978 On the instability of an internal gravity wave. Proc. R. Soc. Lond. A 356, $411-432$.

Gama, S., Vergassola, M. \& Frisch, U. 1994 Negative eddy viscosity in isotropically forced 2-dimensional flow - linear and nonlinear dynamics. J. Fluid Mech. 260, 95-126.

Goldreich, P. \& Schubert, G. 1967 Differential rotation in stars. Astrophys. J. 150, 571-587.

Holford, J. \& Linden, P. F. 1999 Turbulent mixing in a stratified fluid. Dyn. Atmos. Oceans 30, 173-198.

KurgansKy, M. V. 1979 Hydrodynamic instability of internal waves in the atmosphere. Izv. Acad. Sci., USSR, Atmos. Oceanic Phys. 15, 707-710. 
KuRGansky, M. V. 1980 Instability of internal gravity waves propagating at small angles to the vertical. Izv. Acad. Sci., USSR, Atmos. Oceanic Phys. 16, 758-764.

Lombard, P. N. \& Riley, J. J. 1996 Instability and breakdown of internal gravity waves. Phys. Fluids 8, 3271-3287.

Lorenz, E. N. 1972 Barotropic instability of Rossby wave motion. J. Atmos. Sci. 29, 258-269.

MCInTyre, M. E. 1970 Diffusive destabilization of the baroclinic circular vortex. Geophys. Fluid Dyn. 1, 19-57.

Meshalkin, L. \& SinaI, Y. 1961 Investigation of the stability of a stationary solution of a system of equations for the plane movement of an incompressible viscous fluid. J. of Appl. Math. Mech. 25, 1700.

Park, Y.-G., Whitehead, J. \& Gnanadeskian, A. 1994 Turbulent mixing in stratified fluids: layer formation and energetics. J. Fluid Mech. 279, 279-311.

Phillips, O. M. 1972 Turbulence in a strongly stratified fluid - is it unstable? Deep Sea Res. 19, 79-81.

Posmentier, E. S. 1977 The generation of salinity fine structure by vertical diffusion. J. Phys. Oceanogr. 7, 298-300.

Radko, T. 2003 A mechanism for layer formation in a double-diffusive fluid. J. Fluid Mech. 497, $365-380$.

Schmitt, R. W. 1994 Double diffusion in oceanography. Annu. Rev. Fluid Mech. 26, 255-285.

Sivashinsky, G. 1985 Weak turbulence in periodic flows. Physica D 17, 243-255.

THORPE, S. A. 1994 Statically unstable layers produced by overturning internal gravity-waves. J. Fluid Mech. 260, 333-350.

Turner, J. S. 1985 Multicomponent convection. Annu. Rev. Fluid Mech. 17, 11-44.

YIH, C.-S. 1961 Dual role of viscosity in the instability of revolving fluids of variable density. Phys. Fluids 4, 806-811.

Young, Y. 1999 On stratified Kolmogorov flow. Woods Hole Oceanog. Inst. Tech Rep. 40. 